\title{
Subclinical and clinical atherosclerosis in rheumatoid arthritis: results from the 3-year, multicentre, prospective, observational GIRRCS (Gruppo Italiano di Ricerca in Reumatologia Clinica e Sperimentale) study
}

Piero Ruscitti ${ }^{1 *}$ D, Paola Cipriani ${ }^{1}$, Vasiliki Liakouli ${ }^{1}$, Daniela lacono ${ }^{2}$, llenia Pantano ${ }^{2}$, Domenico Paolo Emanuele Margiotta ${ }^{3}$, Luca Navarini ${ }^{3}$, Giulia Maria Destro Castaniti ${ }^{4}$, Nicola Maruotti ${ }^{5}$, Gerardo Di Scala ${ }^{6}$, Licia Picciariello ${ }^{7}$, Francesco Caso ${ }^{8}$, Sara Bongiovanni ${ }^{9}$, Rosa Daniela Grembiale ${ }^{10}$, Fabiola Atzeni ${ }^{11}$, Raffaele Scarpa ${ }^{8}$, Federico Perosa ${ }^{7}$, Giacomo Emmi ${ }^{6}$, Francesco Paolo Cantatore ${ }^{5}$, Giuliana Guggino ${ }^{4}$, Antonella Afeltra ${ }^{3}$, Francesco Ciccia ${ }^{2}$ and Roberto Giacomelli ${ }^{1}$

\begin{abstract}
Background: Rheumatoid arthritis (RA) is associated with an increased risk of morbidity and mortality, when compared with general population, largely due to enhanced atherosclerotic disease. In this work, we aimed at assessing both occurrence and predictive factors of subclinical and clinical atherosclerosis in RA.

Methods: From January 1, 2015, to December 31, 2015, consecutive participants with RA, admitted to Italian Rheumatology Units, were assessed in the GIRRCS (Gruppo Italiano di Ricerca in Reumatologia Clinica e Sperimentale) cohort. After that, those participants were followed up in a 3-year, prospective, observational study, assessing the occurrence of subclinical and clinical atherosclerosis and possible predictive factors. McNemar test was employed to assess the changes in subclinical and clinical atherosclerosis, and regression analyses exploited the ORs for the occurrence of those comorbidities.

Results: We analysed 841 participants, mostly female (82.2\%) and with median age of 60 years (range 21-90). The remission was achieved and maintained by $41.8 \%$ of participants during the follow-up. We observed an increased rate of subclinical atherosclerosis at the end of follow-up (139 vs 203 participants, $p<0.0001$ ), particularly in participants with a disease duration less than 5 years at baseline (70 participants vs 133 participants, $p<0.0001$ ). Type 2 diabetes (T2D) (OR 4.50,95\%Cl 1.74-11.62, $p=0.002)$, high blood pressure (OR 2.03, 95\%Cl 1.04-4.14, $p=$ $0.042)$, ACPA (OR 2.36, 95\%Cl 1.19-4.69, $p=0.014)$ and mean values of CRP during the follow-up (OR $1.07,95 \% \mathrm{Cl}$ $1.03-1.14, p=0.040$ ) were significantly associated with higher risk of subclinical atherosclerosis. We observed an increased rate of clinical atherosclerosis at the end of follow-up (48 vs 76 participants, $p<0.0001$ ). T2D (OR 6.21, $95 \% \mathrm{Cl} 2.19-17.71, p=0.001)$ was associated with a significant risk of clinical atherosclerosis. The achievement and the maintenance of remission reduced the risk of subclinical (OR $0.25,95 \% \mathrm{Cl} 0.11-0.56, p=0.001)$ and clinical atherosclerosis (OR 0.20, 95\% Cl 0.09-0.95, $p=0.041)$.

\footnotetext{
*Correspondence: pieroruscitti@live.com; piero.ruscitti@univaq.it

'Rheumatology Unit; Department of Biotechnological and Applied Clinical

Sciences, University of L'Aquila, delta 6 building, PO box 67100, L'Aquila, Italy

Full list of author information is available at the end of the article
}

(c) The Author(s). 2019 Open Access This article is distributed under the terms of the Creative Commons Attribution 4.0 International License (http://creativecommons.org/licenses/by/4.0/), which permits unrestricted use, distribution, and reproduction in any medium, provided you give appropriate credit to the original author(s) and the source, provide a link to the Creative Commons license, and indicate if changes were made. The Creative Commons Public Domain Dedication waiver (http://creativecommons.org/publicdomain/zero/1.0/) applies to the data made available in this article, unless otherwise stated. 


\begin{abstract}
(Continued from previous page)
Conclusions: We reported an increased prevalence and incidence of both subclinical and clinical atherosclerosis in 3-year prospectively followed participants, mainly in the subset with a duration of disease less than 5 years. The achievement and the maintenance of remission are associated with a reduction of the risk of subclinical and clinical atherosclerosis. Among "traditional" cardiovascular risk factors, participants with T2D showed a higher risk of clinical and subclinical atherosclerosis.
\end{abstract}

Keywords: Rheumatoid arthritis, Atherosclerosis, Cardiovascular diseases, Remission, Type 2 diabetes

\section{Background}

Rheumatoid arthritis (RA) is a systemic, inflammatory disease leading to joint damage and to a reduction of quality of life $[1,2]$. RA is associated with an increased risk of morbidity and mortality, when compared with general population, largely due to enhanced atherosclerotic disease [3, 4]. Despite the improved long-term joint damage by treatment with synthetic and biologic disease-modifying anti-rheumatic drugs (DMARDs) [5-7], the close relationship between $\mathrm{RA}$ and cardiovascular $(\mathrm{CV})$ events has been reported, including myocardial infarction, cerebrovascular accident and congestive heart failure $[8,9]$. Furthermore, the prevalence of subclinical atherosclerosis is increased in RA, as shown by studies assessing the rate of carotid artery plaques $[10$, 11]. Subclinical atherosclerosis may additionally identify those patients with a higher risk to develop CV events [12]. On these bases, multiple lines of research are focused on the development of subclinical and clinical atherosclerosis in RA [13, 14]. This typical clinical phenotype could result from the synergy between the elevated prevalence of "traditional" cardiovascular $(\mathrm{CV})$ risk factors and inflammation [15]. In fact, although the European League Against Rheumatism (EULAR) provided recommendations for the management of CV risk in inflammatory arthritis [16], traditional CV risk factors remain to be underdiagnosed and undertreated in RA, thus contributing to the development of atherosclerotic disease [17]. Concerning the rheumatoid inflammatory process, abundant evidence strongly supports the hypothesis that inflammation could contribute to the pathogenesis of atherosclerotic disease beyond the elevated traditional CV risk factors $[18,19]$. Some well-known pathogenic pro-inflammatory RA mediators could play a pivotal role in the development of atherosclerosis, as suggested by both pre-clinical and clinical reports [20, 21]. In this context, it must be pointed out that the evidence deriving from randomised clinical trials does not entirely clarify this issue. In fact, given strict enrolment criteria, the participants, who are usually enrolled, could not fully mirror the real-life scenario, thus decreasing the generalisability of the results [22]. Furthermore, the evaluation of CV burden is mainly obtained from low-quality studies, generally with a retrospective design and affected by different biases impairing both interpretation and generalisation of results. In fact, few prospective studies in this setting have been planned and performed so far. Finally, a comprehensive evaluation of CV burden in the RA could be complex as well as timeconsuming, and thus, the identification of biomarkers, accurately reflecting this issue, is still awaited $[15,16,19,20$, 23].

In this work, we aimed at evaluating both the occurrence and the predictive factors of subclinical and clinical atherosclerosis in participants with RA, in a 3-year, multicentre, prospective, observational study.

\section{Methods}

\section{Study design}

From January 1, 2015, to December 31, 2015, consecutive participants with RA, admitted to Italian Rheumatology Units, were assessed in the GIRRCS (Gruppo Italiano di Ricerca in Reumatologia Clinica e Sperimentale) cohort [24]. After that, those participants were followed up in a 3year, prospective, observational study, assessing the occurrence of subclinical and clinical atherosclerosis and possible predictive factors. The local Ethics Committee (Comitato Etico Azienda Sanitaria Locale 1 Avezzano/Sulmona/ L'Aquila, L'Aquila, Italy; protocol number 000331/17) approved the study, which was performed according to the Good Clinical Practice guidelines and the Declaration of Helsinki. Informed consent was obtained from each participant for the use of clinical and laboratory data for purposes of the study. In reporting the results, we followed the STROBE checklist (Additional file 1: Table S1).

\section{Setting}

Participants were selected among those attending Rheumatologic Units of GIRRCS, throughout Italy. All the units were characterised by experience in management of RA as well as in observational studies and by high-volume outpatient clinics. Data of participants, who were followed up at least for 3 years, were recorded during the scheduled visits, at baseline, after 12 and 36 months.

\section{Participants}

Participants fulfilling the ACR/EULAR criteria for RA $[25,26]$ were included in the study. 


\section{Variables to be assessed}

The main outcome of the study was the occurrence of subclinical and clinical atherosclerosis in participants with RA, at the end of 3-year prospective follow-up. Subclinical atherosclerosis was defined as the presence of carotid and/or peripheral arteries atherosclerotic lesions detected by ultrasound imaging [27]. Clinical atherosclerosis was defined as the presence of one of the following: myocardial infarction, congestive heart failure, cerebrovascular disease including transitory ischemic attack and/or stroke and clinically relevant peripheral artery disease. The investigators verified history and occurrence of clinical atherosclerosis by review of clinical charts, interview and medical examinations of participants. Following the recent recommendations, about $\mathrm{CV}$ disease (CVD) risk assessment to be performed at least once every 5 years [16], we stratified the participants in $<5$ and $>5$ years of disease duration assessing the main outcomes accordingly. Additionally, by using the JBS3 calculator [28], the 10-year CVD risk was estimated and stratified in 5 categories of risk, $<10 \%$ of 10-year CVD risk, $>10 \%<20 \%$ of 10 -year CVD risk, $>20 \%<30 \%$ of 10 -year CVD risk, $>30 \%<40 \%$ of 10 -year CVD risk and $>40 \%$ of 10 -year CVD risk. Furthermore, predictive factors of these comorbidities were assessed by evaluating two main areas of $\mathrm{CV}$ risk factors, namely the traditional and the "RA-related" ones. Among traditional CV risk factors, we assessed gender, age, smoking habit, body mass index (BMI), high cholesterol, metabolic syndrome (MetS), type 2 diabetes (T2D) and high blood pressure (HBP). BMI was calculated according to the standard formula weight $(\mathrm{kg}) /$ height $(\mathrm{m})^{2}$. High cholesterol was defined as cholesterol $>240 \mathrm{mg} / \mathrm{dl}$ and/or treatment with medications lowering the blood cholesterol levels. MetS, T2D and HBP were defined according to standard criteria and/or treatment with anti-diabetic and/or anti-hypertensive medications [29-31]. Among RA-related risk factors, we assessed the presence of rheumatoid factor (RF) or anti-citrullinated peptide antibodies (ACPA), disease duration, extra-articular features, values of erythrocyte sedimentation rate $(\mathrm{ESR}, \mathrm{mm} / \mathrm{h})$ and $\mathrm{C}$-reactive protein $(\mathrm{CRP})$, radiographic damage, joint surgery, disease activity and remission. Disease duration was assessed from the first disease symptom at the study beginning. Extra-articular features were defined as reported in previous study [32]. Radiographic damage was defined as the presence of at least one marginal erosion on previously performed hand radiography. Disease Activity Score including 28 joints (DAS28-ESR) was used to assess the disease activity and the remission state (DAS28-ESR < 2.6), as previously defined [33]. ESR was included in the assessment of DAS28 in order to maintain the independence of CRP, a well-established $\mathrm{CV}$ risk factor in the general population, for purpose of data analysis. Participants in remission were defined as those reaching after 12 months and maintaining after 36 months a value of DAS28-ESR $<2.6$.

We recorded the administered therapeutic strategies during the follow-up. For those participants who underwent sequential treatment with synthetic or biologic DMARDs, we assigned the treatment category according to the medication to which the participant was exposed for a longer period. Corticosteroids (CCSs) treatment was codified in categories, high dosage and low dosage, as previously identified [34]. We defined participants treated with high dosage of CCSs as those taking $>7.5$ mg prednisone-equivalent for 3 months or more during the observation period, whereas participants treated with low dosage of CCSs as those taking $\leq 7.5 \mathrm{mg}$ prednisoneequivalent. We also recorded the use of aspirin (ASA) during the follow-up.

\section{Data sources}

Relevant data were collected at study beginning and reassessed after 12 and 36 months, during the scheduled visits for each involved participant by an extensive clinical history.

\section{Bias}

Considering the observational design, our study could be subjected to a number of possible biases. We tried to minimise the main methodological problems by a careful definition of each variable to be assessed. Furthermore, participants with significant missing data, which were considered to be meaningful for the analyses, were removed. Specifically, participants with one or more missing data in the main outcomes were removed from the analyses.

\section{Study size}

We would provide a "real-life" estimation of the occurrence of subclinical and clinical atherosclerosis in consecutive participants with RA in Italian Rheumatology Units. From January 1, 2015, to December 31, 2015, consecutive participants with RA, admitted to Italian Rheumatology Units, were assessed and followed up in a 3-year, prospective, observational study.

\section{Statistical methods}

Statistics firstly provided descriptive analysis of the data. Normally distributed continuous variables were expressed as mean \pm standard deviation (SD), otherwise as median and range interquartile, as appropriate. McNemar test was employed to assess the changes in subclinical and clinical atherosclerosis comparing the 
beginning and the end of follow-up and different subsets of participants. Incident cases were reported as incidence proportion and incidence rate per 1000 person-years at risk. Regression analyses exploited the ORs for the occurrence of those comorbidities, considering data at 36 months. The purposeful selection process of covariates started by a univariate analysis of each variable; any variable having a significant univariate test was selected as a possible candidate for the multivariate analyses. Conversely, covariates were removed from the models if non-significant. At the end of this process of deleting and refitting, the multivariate models were built and OR estimations of significant associations with subclinical and clinical atherosclerosis were provided. Multicollinearity was evaluated by using the variance inflation factor (VIF) before entering each variable in regression models. Participants characterised by missing data in main outcomes were excluded from the analysis. Twosided $P$ values $<0.05$ were considered statistically significant. The Statistics Package for Social Sciences (SPSS for Windows, version 17.0, SPSS Inc., Chicago, IL, USA) was used for all analyses.

\section{Results}

\section{Participants and descriptive data}

After assessment of 886 participants, 841 participants with 3 years of prospective follow-up were analysed. In the present evaluation, only participants fully followed up for 3 years were assessed, follow-up at 12 and 36 months was performed in all these participants. The participants, who were excluded, were characterised by missing data in main outcomes. Baseline characteristics of participants are described elsewhere [24]. Briefly, assessed participants were mostly female $(82.2 \%)$, median age of 60 years (range 21-90), median disease duration of 8.20 years (range $0.1-35$ ), $73.1 \%$ displayed the positivity for RF and/or for ACPA. During the follow-up, $72.8 \%$ of the participants were treated with CCSs (mainly at low dosage, $60.0 \%$ of participants), $85.1 \%$ with methotrexate (MTX), and 61.5\% with biologic DMARDs. Concerning the clinical response, we observed that $41.8 \%$ of participants reached and maintained the remission during the follow-up. Regarding traditional CV risk factors, $31.6 \%$ of the participants reported smoking habit, $49.3 \%$ were affected by HBP, $32.1 \%$ by high levels of cholesterol, $22.3 \%$ by MetS and $12.1 \%$ by T2D, as shown in Table 1.

\section{Occurrence of subclinical atherosclerosis}

We recorded that $24.1 \%$ [21.3-26.7] of participants were defined as having subclinical atherosclerosis at the end of follow-up, an increased rate when compared with the beginning of the study (139 participants vs 203 participants, $p<0.0001)$. All participants included in the present evaluation underwent ultrasound of peripheral arteries, which was performed at baseline, after 12 and 36 months. Analysing the incident cases of subclinical atherosclerosis, we estimated an incidence proportion of $10.1 \%$ [8.0-12.0] and, considering over 2010 personyears, an incidence rate of $31.8 \times 1000$ [24.8-38.8] person-years. Furthermore, we analysed these results according to the duration of the disease, stratifying the participants based on duration of disease $<5$ years or duration of disease $>5$ years (Fig. 1). In participants with duration of disease $<5$ years, we recorded an increased rate of subclinical atherosclerosis when compared with participants with duration of disease $>5$ years (70 participants vs 133 participants, $p<0.0001)$. Analysing the incident cases of subclinical atherosclerosis in these subsets, we estimated an incidence proportion of $20.7 \%$ [18.1-23.3] and, considering over 610 person-years, an incidence rate of $77.1 \times 1000$ person-years [56.6-97.6] in participants with duration of disease $<5$ years. Conversely, in participants with duration of disease $>5$ years, we estimated an incidence proportion of 5.6\% [3.4-7.8] and, considering over 1149 person-years, an incidence rate of $19.1 \times 1000$ person-years [8.4-29.8] in participants with duration of disease $>5$ years.

\section{Predictive factors of subclinical atherosclerosis}

A logistic regression model was built in 697 participants to evaluate the possible predictive role of selected variables (age, male gender, HBP, T2D, ACPA, mean values of CRP during the follow-up, remission) on the likelihood of having subclinical atherosclerosis, after 36 months. The participants, who were excluded, were characterised by baseline evidence of subclinical and/or clinical atherosclerosis. The analysis showed that T2D, HBP, ACPA, remission and mean values of CRP during the follow-up were independently associated with subclinical atherosclerosis. Participants with comorbid T2D (OR 4.50, 95\%CI 1.74-11.62, $p=$ 0.002 ) and with comorbid HBP (OR 2.03, 95\%CI 1.04-4.14, $p=0.042$ ) were significantly associated with a higher risk of having subclinical atherosclerosis. The presence of ACPA (OR 2.36, 95\%CI 1.19-4.69, $p=$ $0.014)$ and mean values of CRP during the follow-up (OR $1.07,95 \%$ CI $1.03-1.14, p=0.040$ ) were also significantly associated with a higher risk of that comorbidity. Of interest, the participants reaching and maintaining remission were significantly associated with a reduced risk of having subclinical atherosclerosis (OR $0.25,95 \%$ CI $0.11-0.56, p=0.001$ ), as shown in Table 2. The logistic regression model was statistically significant $\left(\chi^{2}=13.09, p<0.0001\right)$. Regarding the possible predictive role of treatment on subclinical atherosclerosis, we did not retrieve any significant result concerning the possible predictive role of ASA, 
Table 1 Descriptive statistics

\begin{tabular}{|c|c|}
\hline \multicolumn{2}{|l|}{ Clinical variables } \\
\hline Participants, number & 841 \\
\hline \multicolumn{2}{|l|}{ Demographic characteristics } \\
\hline Age (841 participants), median (range) & 60 years $(21-90)$ \\
\hline Female gender (841 participants), $n$ (\%) & $691(82.2 \%)$ \\
\hline \multicolumn{2}{|l|}{ RA-related features } \\
\hline RF and/or ACPA (841 participants), $n$ (\%) & $615(73.1 \%)$ \\
\hline Disease duration (834 participants), median (range) & 8.2 years $(0.1-35)$ \\
\hline Extra-articular features (840 participants), n (\%) & $138(16.4 \%)$ \\
\hline Radiographic damage (814 participants), n (\%) & $383(47.1 \%)$ \\
\hline Joint surgery (841 participants), $n$ (\%) & $101(12.4 \%)$ \\
\hline Maintenance of remission (836 participants), n (\%) & $349(41.8 \%)$ \\
\hline CRP (833 participants), mean \pm SD & $4.31 \pm 3.62 \mathrm{mg} / \mathrm{L}$ \\
\hline \multicolumn{2}{|l|}{ Traditional CV risk factors } \\
\hline BMI (829 participants), mean \pm SD & $27.01 \pm 4.02$ \\
\hline HBP (811 participants), $n$ (\%) & $400(49.3 \%)$ \\
\hline High cholesterol (798 participants), n (\%) & $256(32.1 \%)$ \\
\hline Smoking habit (836 participants), n (\%) & $264(31.6 \%)$ \\
\hline MetS (807 participants), $n$ (\%) & $180(22.3 \%)$ \\
\hline T2D (811 participants), $n$ (\%) & $98(12.1 \%)$ \\
\hline \multicolumn{2}{|l|}{ Therapies } \\
\hline ASA (838 participants), $n$ (\%) & $320(38.2 \%)$ \\
\hline CCS (841 participants), $n$ (\%) & $612(72.8 \%)$ \\
\hline CCSs low dosage (841 participants), $n$ (\%) & $504(60.0 \%)$ \\
\hline MTX (841 participants), $n$ (\%) & $716(85.1 \%)$ \\
\hline HCQ (813 participants), n (\%) & $231(28.5 \%)$ \\
\hline LEF (841 participants), $n$ (\%) & $186(22.1 \%)$ \\
\hline SSZ (841 participants), n (\%) & $113(13.4 \%)$ \\
\hline Biologic DMARDs (841 participants), $n$ (\%) & $517(61.5 \%)$ \\
\hline TNFi (841 participants), $n$ (\%) & $308(36.6 \%)$ \\
\hline Non TNFi (841 participants), n (\%) & $209(24.9 \%)$ \\
\hline
\end{tabular}

Participants with missing data, $n$ (\%): disease duration, 7 (0.8\%); extra-articular features, $1(0.1 \%)$; radiographic damage, 27 (3.2\%); maintenance of remission, $5(0.6 \%) ;$ CRP, 8 (0.9\%); BMI, 12 (1.4\%); high cholesterol, 43 (5.1\%); HBP, 30 (3.6\%); smoking habit, 5 (0.6\%); MetS, 34 (4.0\%); T2D, 30 (3.6\%); ASA, 3 (0.3\%); $\mathrm{HCQ}, 28(3.3 \%)$

Abbreviations: $R A$ rheumatoid arthritis, $R F$ rheumatoid factor, $A C P A$ Anticitrullinated protein antibodies, $C R P$ mean values of $C$ reactive protein during the follow-up, $S D$ standard deviation, $C V$ cardiovascular, $B M I$ mean body mass index during the follow-up, MetS metabolic syndrome, HBP high blood pressure, T2D type 2 diabetes, ASA acetylsalicylic acid, aspirin, CCSs corticosteroids, MTX methotrexate, HCQ hydroxychloroquine, SSZ sulfasalazine, LEF leflunomide, TNFi tumour necrosis factor inhibitor

MTX, HCQ, low dosage CCSs, high dosage CCSs, TNFi and non-TNFi.

\section{Occurrence of clinical atherosclerosis}

We observed that 9.0\% [8.8-9.2] of participants were defined as having clinical atherosclerosis at the end of the follow-up, an increased rate when compared with the beginning of the study (48 participants vs 76 participants, $p<0.0001)$. Analysing the incident cases of clinical atherosclerosis, we estimated an incidence proportion of $3.6 \%$ [2.4-4.8] and, considering over 2337 person-years, an incidence rate of $12.0 \times 1000$ person-years [8.0-16.0]. Furthermore, we analysed these results according to the duration of the disease, stratifying the participants based on duration of disease $<5$ years or duration of disease $>5$ years (Fig. 1). In participants with duration of disease $<5$ years, we recorded an increased rate of clinical atherosclerosis when compared with participants with duration of disease $>5$ years (27 participants vs 49 participants, $p<0.0001)$. Analysing the incident cases of clinical atherosclerosis in these subsets, we estimated an incidence proportion of $19.5 \%$ [14.8-24.1] and, considering over 832 person-years, an incidence rate of $32.4 \times$ 1000 person-years [20.4-44.4] in participants with duration of disease $<5$ years. Conversely, in participants with duration of disease $>5$ years, we estimated an incidence proportion of $0.5 \%[0.2-0.8]$ and, considering over 1248 person-years, an incidence rate of $1.6 \times 1000$ person-years [0.6-3.8] in participants with duration of disease $>5$ years.

In 697 participants without baseline evidence of subclinical and clinical atherosclerosis, the 10-year CVD risk was estimated. We retrieved that 267 participants $(38.4 \%)$ displayed $<10 \%$ of 10 -year CVD risk, $249(40.0 \%)<10 \%<20 \%$ of 10-year CVD risk, 86 $(12.3 \%)>20 \%<30 \%$ of 10 -year CVD risk, $43(6.2 \%)$ $>30 \%<40 \%$ of 10 -year CVD risk and $22(3.1 \%)>$ $40 \%$ of 10 -year CVD risk.

\section{Predictive factors of clinical atherosclerosis}

A logistic regression model was built in 697 participants to evaluate the possible predictive role of selected variables (age, male gender, HBP, T2D, remission) on the likelihood of having clinical atherosclerosis. The participants, who were excluded, were characterised by baseline evidence of subclinical and/or clinical atherosclerosis. The analysis showed that T2D and remission were independently associated with subclinical atherosclerosis. Participants with comorbid T2D (OR 6.21, 95\%CI 2.19-17.71, $p=0.001$ ) were associated with a higher risk of having clinical atherosclerosis. Conversely, the participants reaching and maintaining remission were associated with a reduced risk of clinical atherosclerosis (OR $0.20,95 \%$ CI $0.09-0.95, p=$ 0.041 ), as shown in Table 3. The logistic regression model was statistically significant $\left(x^{2}=6.37, p=0.012\right)$. As reported for subclinical atherosclerosis, we did not retrieve any significant result concerning the possible predictive role of ASA, MTX, HCQ, low dosage CCSs, high dosage CCSs, TNFi and non-TNFi on clinical atherosclerosis. 


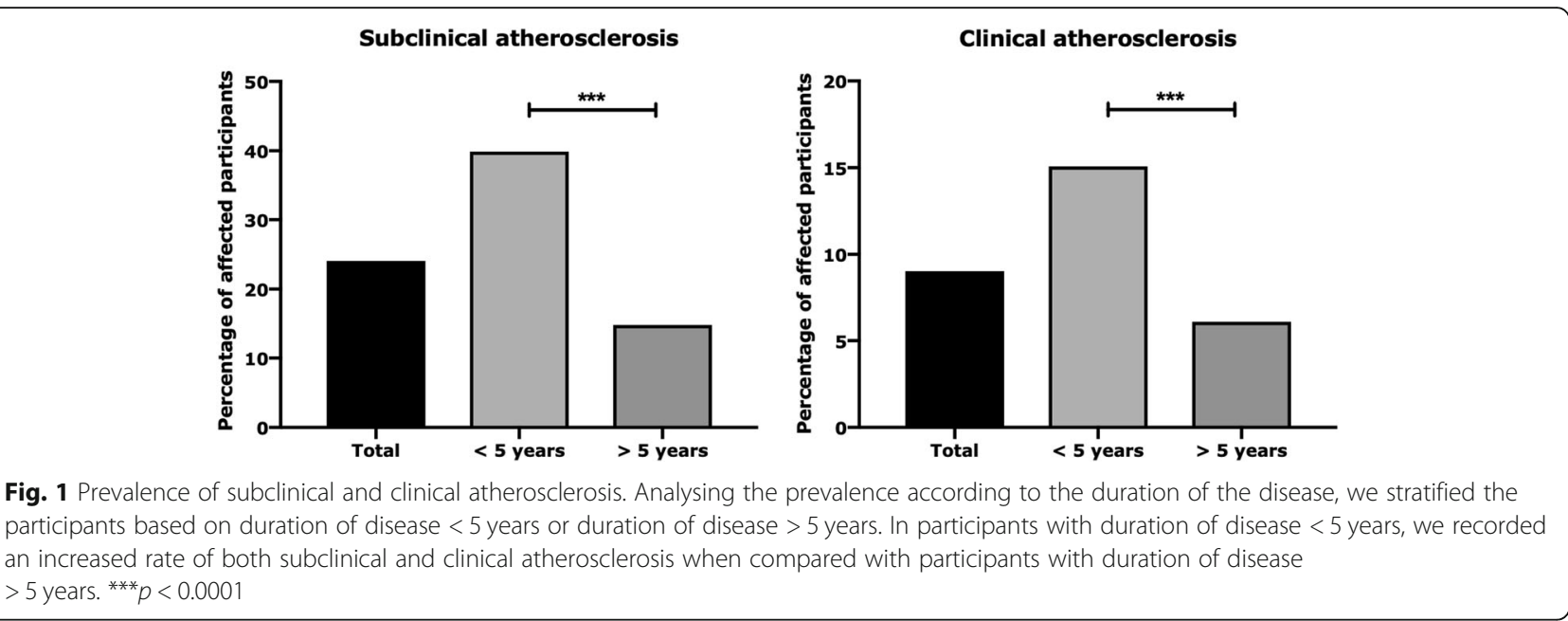

\section{Discussion}

Although the increased CV risk has been established since many years in RA, only few prospective studies in this setting have been planned and performed. Thus, we designed a 3 -year prospective study, enrolling a large cohort of participants, in order to provide a real-life estimation of the $\mathrm{CV}$ burden in RA. We reported an increased prevalence and incidence of both subclinical and clinical atherosclerosis in prospectively followed participants, mainly in the subset with a duration of disease less than 5 years, suggesting the role of active inflammatory process. We also showed that the occurrence of subclinical and clinical atherosclerosis derives from a synergy between both RA-related and traditional CV risk factors, pointing out the need of a strict management of both systemic inflammation and $\mathrm{CV}$ risk factors to improve the long-term outcome of RA.

In our study, we observed a lower occurrence of subclinical and clinical atherosclerosis than reported in available meta-analyses on these topics $[3,4,10]$. However, our results showed a noteworthy increased prevalence and incidence of both subclinical and clinical atherosclerosis in participants with a duration of disease less than 5 years. The decreased prevalence of both subclinical and clinical atherosclerosis observed in our participants with longer disease duration cannot be attributed to deaths or participants lost to follow-up; due to the specific design of our study, we analysed only participants fully completing 3 years of prospective follow-up. Furthermore, these results could suggest that the increased risk of subclinical and clinical atherosclerosis is already present in the early stages of RA [35, 36], and not only related to the reported accrual CV damage $[3,4,10]$. In this context, patients with RA could be more prone to plaque instability and rupture, in addition to accelerated atherosclerosis [9]. The inflammatory process could contribute more specifically to more severe acute coronary syndromes and strokes [37], which may be more strongly associated with the presence and severity of local or systemic inflammation than with disease duration [38]. In addition, some reversals of the vascular damage could be achieved after anti-inflammatory therapies $[39,40]$, suggesting the long-term improvement of $\mathrm{CV}$ burden observed in our study. However, considering the evidence of some conflicting results concerning the role of duration of disease on accelerated atherosclerosis in RA [41], further specifically designed studies are necessary to entirely elucidate this issue of CV burden in early stages of the disease.

Analysing predictive factors of occurrence, we clearly showed that remission significantly reduced the risk of both subclinical and clinical atherosclerosis. We observed that participants reaching and maintaining the remission during the follow-up experienced a lesser risk of having these comorbidities. Our data suggest that the inflammatory process may strongly contribute in enhancing the CV risk $[14,15,42]$ and the remission ought to be considered the pivotal goal for $\mathrm{CV}$ risk management in patients with RA [43]. In fact, a treat-to-target intervention, aiming at remission, showed to significantly reduce the occurrence of clinical and subclinical atherosclerosis in a clinical trial $[16,44]$. As suggested by our data, the consequent decline of CV burden in RA could be attributable to this better disease management $[13,45]$. In fact, the delay of diagnosis and therapy of RA could enhance the clinical atherosclerosis, supporting the importance of early recognition and treatment of these patients [46]. Assessing further predictive factors among the RA-related CV risk factors, we reported an association between ACPA positivity and elevated CRP levels with subclinical atherosclerosis. In fact, patients affected by a seropositive disease with high inflammatory burden experience an accelerated atherosclerosis [47]. Our prospective study confirms previous paper reporting the ACPA positivity as a risk factor for development of 
Table 2 Regression analyses assessing predictive factors of subclinical atherosclerosis

\begin{tabular}{|c|c|c|c|}
\hline Clinical variables & OR & $95 \% \mathrm{Cl}$ & $p$ \\
\hline \multicolumn{4}{|l|}{ Univariate analyses } \\
\hline Age & 1.01 & $0.99-1.02$ & 0.10 \\
\hline Male gender & 1.18 & $0.79-1.76$ & 0.42 \\
\hline RF & 1.15 & $0.81-1.65$ & 0.43 \\
\hline ACPA & 1.40 & $1.01-1.94$ & 0.042 \\
\hline Disease duration & 1.00 & $0.98-1.02$ & 0.99 \\
\hline Extra-articular features & 1.31 & $0.74-1.72$ & 0.56 \\
\hline Radiographic damage & 0.85 & $0.61-1.16$ & 0.30 \\
\hline Joint surgery & 1.32 & $0.83-2.08$ & 0.23 \\
\hline Maintenance of remission & 0.65 & $0.47-0.91$ & 0.014 \\
\hline CRP & 1.04 & $1.02-1.07$ & 0.002 \\
\hline BMI & 1.02 & $0.89-1.08$ & 0.52 \\
\hline $\mathrm{HBP}$ & 8.79 & $5.87-13.18$ & $<0.0001$ \\
\hline High cholesterol & 2.32 & $0.89-5.53$ & 0.89 \\
\hline Smoking habit & 0.99 & $0.71-1.40$ & 0.99 \\
\hline MetS & 3.99 & $2.83-5.64$ & $<0.0001$ \\
\hline $\mathrm{T} 2 \mathrm{D}$ & 3.16 & $2.04-4.88$ & $<0.0001$ \\
\hline ASA & 2.59 & $0.98-4.13$ & 0.08 \\
\hline CCS & 1.36 & $0.68-2.04$ & 0.78 \\
\hline CCSs low dosage & 1.25 & $0.87-1.78$ & 0.22 \\
\hline MTX & 1.67 & $0.98-2.75$ & 0.08 \\
\hline $\mathrm{HCQ}$ & 0.62 & $0.42-1.01$ & 0.09 \\
\hline LEF & 0.73 & $0.49-1.09$ & 0.13 \\
\hline SSZ & 0.98 & $0.61-1.57$ & 0.95 \\
\hline Biologic DMARDs & 0.58 & $0.42-1.01$ & 0.07 \\
\hline TNFi & 0.99 & $0.71-1.37$ & 0.95 \\
\hline Non-TNFi & 0.67 & $0.24-1.02$ & 0.12 \\
\hline \multicolumn{4}{|l|}{ Multivariate analysis } \\
\hline Age & 1.20 & $0.99-1.05$ & 0.13 \\
\hline Male gender & 1.29 & $0.55-3.04$ & 0.55 \\
\hline $\mathrm{HBP}$ & 2.03 & $1.04-4.14$ & 0.042 \\
\hline $\mathrm{T} 2 \mathrm{D}$ & 4.50 & $1.74-11.62$ & 0.002 \\
\hline ACPA & 2.36 & $1.19-4.69$ & 0.014 \\
\hline CRP & 1.07 & $1.03-1.14$ & 0.040 \\
\hline Remission & 0.25 & $0.11-0.56$ & 0.001 \\
\hline
\end{tabular}

Italicised values are statistically significant $(p<0.05)$

Abbreviations: $R F$ rheumatoid factor, $A C P A$ Anti-citrullinated protein antibodies, $C R P$ mean values of $C$ reactive protein during the follow-up, $B M I$ mean body mass index during the follow-up, MetS metabolic syndrome, $\mathrm{HBP}$ high blood pressure, T2D type 2 diabetes, ASA acetylsalicylic acid, aspirin, CCSs

corticosteroids, MTX methotrexate, HCQ hydroxychloroquine, SSZ sulfasalazine, LEF leflunomide, TNFi tumour necrosis factor inhibitor

atherosclerosis in RA, independently of traditional ones [48]. It has been also suggested that ACPA could impair the resolution of inflammation within the atherosclerotic plaque, enhancing the evolution and destabilisation of
Table 3 Regression analyses assessing predictive factors of clinical atherosclerosis

\begin{tabular}{|c|c|c|c|}
\hline Clinical variables & OR & $95 \% \mathrm{Cl}$ & p \\
\hline \multicolumn{4}{|l|}{ Univariate analyses } \\
\hline Age & 1.01 & $0.99-1.03$ & 0.30 \\
\hline Male gender & 1.14 & $0.63-2.08$ & 0.65 \\
\hline RF & 1.33 & $0.77-2.32$ & 0.31 \\
\hline ACPA & 0.73 & $0.45-1.28$ & 0.20 \\
\hline Disease duration & 0.99 & $0.95-1.02$ & 0.58 \\
\hline Extra-articular features & 1.67 & $0.94-2.93$ & 0.08 \\
\hline Radiographic damage & 089 & $0.55-1.43$ & 0.63 \\
\hline Joint surgery & 0.82 & $0.38-1.76$ & 0.61 \\
\hline Maintenance of remission & 0.55 & $0.34-0.91$ & 0.20 \\
\hline CRP & 1.02 & $0.99-1.05$ & 0.14 \\
\hline BMl & 1.05 & $0.99-1.08$ & 0.11 \\
\hline $\mathrm{HBP}$ & 10.69 & $5.06-22.59$ & $<0.0001$ \\
\hline High cholesterol & 2.47 & $0.56-5.9$ & 0.18 \\
\hline Smoking habit & 0.80 & $0.47-1.35$ & 0.40 \\
\hline MetS & 6.27 & $3.79-10.37$ & $<0.0001$ \\
\hline $\mathrm{T} 2 \mathrm{D}$ & 2.64 & $1.48-4.71$ & 0.001 \\
\hline ASA & 1.11 & $0.78-2.01$ & 0.85 \\
\hline CCS & 1.44 & 0.81 & 2.56 \\
\hline CCSs low dosage & 1.91 & $0.89-3.46$ & 0.09 \\
\hline MTX & 1.31 & $0.64-2.72$ & 0.45 \\
\hline $\mathrm{HCQ}$ & 0.61 & $0.34-1.08$ & 0.09 \\
\hline LEF & 0.51 & $0.25-1.07$ & 0.07 \\
\hline SSZ & 0.53 & $0.23-1.24$ & 0.14 \\
\hline Biologic DMARDs & 0.67 & $0.29-1.36$ & 0.35 \\
\hline TNFi & 0.55 & $0.32-1.01$ & 0.07 \\
\hline Non-TNFi & 0.59 & $0.31-1.02$ & 0.09 \\
\hline \multicolumn{4}{|l|}{ Multivariate analysis } \\
\hline Age & 1.01 & $0.99-1.05$ & 0.44 \\
\hline Male gender & 0.62 & $0.22-1.73$ & 0.36 \\
\hline $\mathrm{HBP}$ & 1.85 & $0.69-4.91$ & 0.21 \\
\hline $\mathrm{T} 2 \mathrm{D}$ & 6.21 & $2.19-17.71$ & 0.001 \\
\hline Remission & 0.20 & $0.09-0.95$ & 0.041 \\
\hline
\end{tabular}

Italicised values are statistically significant $(p<0.05)$

Abbreviations: $R F$ rheumatoid factor, ACPA Anti-citrullinated protein antibodies, $C R P$ mean values of $C$ reactive protein during the follow-up, $B M I$ mean body mass index during the follow-up, MetS metabolic syndrome, HBP high blood pressure, T2D type 2 diabetes, ASA acetylsalicylic acid, aspirin, CCSS corticosteroids, MTX methotrexate, HCQ hydroxychloroquine, SSZ sulfasalazine, LEF leflunomide, TNFi tumour necrosis factor inhibitor

the lesions $[19,49]$. In addition, ACPA positivity could correlate with vascular calcifications in RA, a further marker of accelerated atherosclerosis [50].

Analysing traditional CV risk factors, we observed that participants with comorbid T2D were at higher risk of both subclinical and clinical atherosclerosis. Insulin 
resistance and T2D were shown as being highly prevalent in RA and to enhance the CV burden [51, 52]. Furthermore, T2D and RA could share pathogenic inflammatory pathways suggesting possible common therapeutic targets [53-55]. We also observed that participants with comorbid HBP were associated with enhanced risk of subclinical atherosclerosis. In fact, HBP was reported as being highly prevalent in RA, and it is considered one of the most important predictor of atherosclerosis in rheumatic diseases [56]. Conversely, in our analyses, some well-known traditional CV risk factors were not associated with subclinical and clinical atherosclerosis, including BMI, cholesterol and smoking habit. Our study confirms what already reported about some RA paradoxes in the analysis of CV risk. In fact, the rheumatoid sarcopenia, altering the body composition, impairs the predictive role of BMI on atherosclerotic disease, and the qualitative changes of lipoproteins induced by the rheumatoid process cannot be routinely assessed by the quantitative tests, thus limiting the predictive role of dyslipidaemia in these participants [57, 58]. Finally, the "smoking paradox" was described in RA due to an index event bias, since smoking habit is associated with RA and with its associated comorbidities [59].

The results of this study did not show the predictive role of administered therapies on the main outcomes. In fact, based on the real-life design of the study, the therapies were not systematically administered, and the choice of medications was left to the physicians, with the consequent risk of a "confounding by indication" bias, due to the possibility that more intensive treatment could be administered to those participants affected by a more aggressive disease. In this context, the lack of randomised controlled trials, specifically designed to evaluate the effect of different drugs in controlling the insurgence of atherosclerotic disease in RA, could impair the possibility to reach definitive conclusions.

Our study could be affected by different limitations, reducing the external validity of the results and suggesting a cautious interpretation. Despite providing an insight into CV risk associated with RA, the lack of a control group avoided to quantify the relative risk of new-onset subclinical and clinical atherosclerosis when compared with matched participants from general population. Furthermore, the lack of data regarding aortic atherosclerosis, thoracic or abdominal aortic aneurysm and angina pectoris could be considered a further limitation of the present work. In addition, the original study design did not allow to fully ascertain the role of therapeutic strategies on subclinical and clinical atherosclerosis. Further studies, specifically designed and adequately powered, are needed to fully elucidate the CV burden in RA and the best therapeutic strategy of those comorbidities and to confirm the results of the more recent diagnostic techniques and biomarkers in CV assessment in RA [60-62]. Finally, the management of missing data by listwise deletion, removing all data for an observation that has one or more missing values, could also be disadvantageous than imputation methods, such as last observation carried forward. In fact, although we considered of not having inadvertently removed a class of participants and that our sample could be large enough to drop data without substantial loss of statistical power, the assumptions of "missing completely at random" could be typically difficult to support, producing possible biased estimates.

\section{Conclusions}

In conclusion, we reported an increased prevalence and incidence of both subclinical and clinical atherosclerosis in prospectively followed participants, mainly in the subset with a duration of disease less than 5 years. We observed that achievement and maintenance of remission is associated with a reduction of subclinical and clinical atherosclerosis in prospectively followed participants. Among traditional CV risk factors, T2D was significantly associated with both subclinical and clinical atherosclerosis, while about other traditional CV risk factors the RA-specific paradoxes may limit the role of tests assessing the lipid profile, smoking habit and BMI. Taking together, our data support the need of a multi-expertise management of RA, counteracting the synergy between the systemic inflammatory process and the traditional $\mathrm{CV}$ risk factors in reducing the $\mathrm{CV}$ burden and, thus, improving the long-term outcome of patients with RA.

\section{Additional file}

Additional file 1: STROBE 2007 (v4) checklist of items to be included in reports of observational studies in epidemiology* (DOC 96 kb)

\section{Abbreviations}

RA: Rheumatoid arthritis; DMARDs: Disease-modifying anti-rheumatic drugs; CV: Cardiovascular; EULAR: European League Against Rheumatism; GIRRCS: Gruppo Italiano di Ricerca in Reumatologia Clinica e Sperimentale; BMI: Body mass index; MetS: Metabolic syndrome; T2D: Type 2 diabetes; HBP: High blood pressure; RF: Rheumatoid factor; ACPA: Anti-citrullinated peptide antibodies; ESR: Erythrocyte sedimentation rate; CRP: C-reactive protein; DAS28: Disease Activity Score including 28 joints;

CCSs: Corticosteroids; ASA: Aspirin; SD: Standard deviation; VIF: Variance inflation factor; MTX: Methotrexate; HCQ: Hydroxychloroquine; TNFi: Tumour necrosis factor inhibitor

\section{Acknowledgements}

The authors thank Mrs. Federica Sensini for her technical assistance.

\section{Authors' contributions}

All authors made substantial contributions to the conception or design of the work and the acquisition and interpretation of data. All authors contributed to the critical review and revision of the manuscript and approved the final version. All the authors agreed to be accountable for all aspects of the work. 


\section{Funding}

No funding for this study.

\section{Availability of data and materials}

All data relevant to the study are included in the article or uploaded as supplementary information.

\section{Ethics approval and consent to participate}

The local Ethics Committee (Comitato Etico Azienda Sanitaria Locale 1 Avezzano/Sulmona/L'Aquila, L'Aquila, Italy; protocol number 000331/17) approved the study, which was performed according to the Good Clinical Practice guidelines and the Declaration of Helsinki. Informed consent was obtained from each participant for the use of clinical and laboratory data for study purposes.

\section{Consent for publication}

Not applicable, all the participants' data are de-identified.

\section{Competing interests}

The authors declare that they have no competing interests.

\section{Author details}

${ }^{1}$ Rheumatology Unit; Department of Biotechnological and Applied Clinical Sciences, University of L'Aquila, delta 6 building, PO box 67100, L'Aquila, Italy. ${ }^{2}$ Department of Clinical and Experimental Medicine, Rheumatology Section, University of Campania "Luigi Vanvitelli", Naples, Italy. ${ }^{3}$ Unit of Allergy, Clinical Immunology and Rheumatology, Department of Medicine, Campus Bio-Medico University of Rome, Rome, Italy. ${ }^{4}$ Department of Health Promotion, Mother and Child Care, Internal Medicine and Medical Specialties (PROMISE), University of Palermo, Palermo, Italy. ${ }^{5}$ Department of Medical and Surgery Sciences, Rheumatology Unit, University of Foggia, Foggia, Italy. ${ }^{6}$ Department of Experimental and Clinical Medicine, University of Florence, Florence, Italy. ${ }^{7}$ Department of Biomedical Sciences and Human Oncology (DIMO), Rheumatologic and Systemic Autoimmune Diseases Unit, University of Bari Medical School, Bari, Italy. ${ }^{8}$ Rheumatology Unit, Department of Clinical Medicine and Surgery, School of Medicine, University of Naples Federico II, Naples, Italy. ${ }^{9}$ Unità di Reumatologia, Ospedale L. Sacco, Milan, Italy.

${ }^{10}$ Rheumatology Research Unit, Department of Health Sciences, University of Catanzaro 'Magna Graecia', Catanzaro, Italy. ${ }^{11}$ Rheumatology Unit, Department of Clinical and Experimental Medicine, University of Messina, Messina, Italy.

Received: 30 April 2019 Accepted: 9 August 2019 Published online: 03 September 2019

\section{References}

1. Barile A, Arrigoni F, Bruno F, Guglielmi G, Zappia M, Reginelli A, et al. Computed tomography and MR imaging in rheumatoid arthritis. Radiol Clin N Am. 2017:55:997-1007

2. Giacomelli R, Gorla R, Trotta F, Tirri R, Grassi W, Bazzichi L, et al. Quality of life and unmet needs in patients with inflammatory arthropathies: results from the multicentre, observational RAPSODIA study. Rheumatology (Oxford). 2015;54:792-7.

3. Aviña-Zubieta JA, Choi HK, Sadatsafavi M, Etminan M, Esdaile JM, Lacaille D. Risk of cardiovascular mortality in patients with rheumatoid arthritis: a metaanalysis of observational studies. Arthritis Rheum. 2008:59:1690-7.

4. Avina-Zubieta JA, Thomas J, Sadatsafavi M, Lehman AJ, Lacaille D. Risk of incident cardiovascular events in patients with rheumatoid arthritis: a metaanalysis of observational studies. Ann Rheum Dis. 2012:71:1524-9.

5. Smolen JS, Aletaha D, McInnes IB. Rheumatoid arthritis. Lancet. 2016; 388:2023-38

6. Cipriani P, Ruscitti P, Carubbi F, Liakouli V, Giacomelli R. Methotrexate in rheumatoid arthritis: optimizing therapy among different formulations. Current and emerging paradigms. Clin Ther. 2014;36:427-35.

7. Burmester GR, Pope JE. Novel treatment strategies in rheumatoid arthritis. Lancet. 2017:389:2338-48.

8. Nicola PJ, Crowson CS, Maradit-Kremers H, Ballman KV, Roger VL, Jacobsen SJ, et al. Contribution of congestive heart failure and ischemic heart disease to excess mortality in rheumatoid arthritis. Arthritis Rheum. 2006;54:60-7.

9. Maradit-Kremers H, Crowson CS, Nicola PJ, Ballman KV, Roger VL, Jacobsen SJ, et al. Increased unrecognized coronary heart disease and sudden deaths in rheumatoid arthritis: a population-based cohort study. Arthritis Rheum. 2005:52:402-11.

10. Ambrosino P, Lupoli R, Di Minno A, Tasso M, Peluso R, Di Minno MN. Subclinical atherosclerosis in patients with rheumatoid arthritis. A metaanalysis of literature studies. Thromb Haemost. 2015;113:916-30.

11. Wah-Suarez MI, Galarza-Delgado DA, Azpiri-Lopez JR, Colunga-Pedraza IJ, Abundis-Marquez EE, Davila-Jimenez JA, et al. Carotid ultrasound findings in rheumatoid arthritis and control subjects: a case-control study. Int J Rheum Dis. 2019;22:25-31.

12. Inaba Y, Chen JA, Bergmann SR. Carotid plaque, compared with carotid intima-media thickness, more accurately predicts coronary artery disease events: a meta-analysis. Atherosclerosis. 2012;220:128-33.

13. Hollan I, Meroni PL, Ahearn JM, Cohen Tervaert JW, Curran S, Goodyear CS, et al. Cardiovascular disease in autoimmune rheumatic diseases. Autoimmun Rev. 2013:12:1004-15.

14. Romano S, Salustri E, Ruscitti P, Carubbi F, Penco M, Giacomelli R. Cardiovascular and metabolic comorbidities in rheumatoid arthritis. Curr Rheumatol Rep. 2018;20:81

15. Nurmohamed MT, Heslinga M, Kitas GD. Cardiovascular comorbidity in rheumatic diseases. Nat Rev Rheumatol. 2015;11:693-704.

16. Agca R, Heslinga SC, Rollefstad S, Heslinga M, Mclnnes IB, Peters MJ, et al. EULAR recommendations for cardiovascular disease risk management in patients with rheumatoid arthritis and other forms of inflammatory joint disorders: 2015/2016 update. Ann Rheum Dis. 2017;76:17-28.

17. van Breukelen-van der Stoep DF, van Zeben D, Klop B, van de Geijn GJ, Janssen $\mathrm{HJ}$, van der Meulen N, et al. Marked underdiagnosis and undertreatment of hypertension and hypercholesterolaemia in rheumatoid arthritis. Rheumatology (Oxford). 2016;55:1210-6.

18. Suciu CF, Prete M, Ruscitti P, Favoino E, Giacomelli R, Perosa F. Oxidized low density lipoproteins: the bridge between atherosclerosis and autoimmunity. Possible implications in accelerated atherosclerosis and for immune intervention in autoimmune rheumatic disorders. Autoimmun Rev. 2018;17:366-75.

19. Skeoch S, Bruce IN. Atherosclerosis in rheumatoid arthritis: is it all about inflammation? Nat Rev Rheumatol. 2015;11:390-400.

20. England BR, Thiele GM, Anderson DR, Mikuls TR. Increased cardiovascular risk in rheumatoid arthritis: mechanisms and implications. BMJ. 2018;361:k1036.

21. Pingiotti $E$, Cipriani $P$, Marrelli $A$, Liakouli $V$, Fratini $S$, Penco $M$, et al. Surface expression of fractalkine receptor (CX3CR1) on CD4+/CD28 T cells in RA patients and correlation with atherosclerotic damage. Ann N Y Acad Sci. 2007;1107:32-41.

22. Pincus T, Sokka T. Should contemporary rheumatoid arthritis clinical trials be more like standard patient care and vice versa? Ann Rheum Dis. 2004; 63(Suppl 2):ii32-9.

23. Giacomelli R, Afeltra A, Alunno A, Bartoloni-Bocci E, Berardicurti O, Bombardieri $\mathrm{M}$, et al. Guidelines for biomarkers in autoimmune rheumatic diseases - evidence based analysis. Autoimmun Rev. 2019;18:93-106.

24. Ruscitti P, Margiotta DPE, Macaluso F, lacono D, D'Onofrio F, Emmi G, et al. Subclinical atherosclerosis and history of cardiovascular events in Italian patients with rheumatoid arthritis: results from a cross-sectional, multicenter GIRRCS (Gruppo Italiano di Ricerca in Reumatologia Clinica e Sperimentale) study. Medicine (Baltimore). 2017:96:e8180.

25. Aletaha D, Neogi T, Silman AJ, Funovits J, Felson DT, Bingham CO 3rd, et al. 2010 rheumatoid arthritis classification criteria: an American College of Rheumatology/European League Against Rheumatism collaborative initiative. Arthritis Rheum. 2010;62:2569-81.

26. Arnett FC, Edworthy SM, Bloch DA, McShane DJ, Fries JF, Cooper NS, et al. The American Rheumatism Association 1987 revised criteria for the classification of rheumatoid arthritis. Arthritis Rheum. 1988;31:315-24.

27. Touboul PJ, Hennerici MG, Meairs S, Adams H, Amarenco P, Bornstein N, et al. Mannheim carotid intima-media thickness consensus (2004-2006). An update on behalf of the Advisory Board of the 3rd and 4th Watching the Risk Symposium, 13th and 15th European Stroke Conferences, Mannheim, Germany, 2004, and Brussels, Belgium, 2006. Cerebrovasc Dis. 2007;23:75-80.

28. JBS3 Board. Joint British Societies' consensus recommendations for the prevention of cardiovascular disease (JBS3). Heart. 2014;100(Suppl 2):ii1-ii67.

29. Alberti KG, Eckel RH, Grundy SM, Zimmet PZ, Cleeman Jl, Donato KA, et al. Harmonizing the metabolic syndrome: a joint interim statement of the International Diabetes Federation Task Force on Epidemiology and Prevention; National Heart, Lung, and Blood Institute; American Heart Association; World 
Heart Federation; International Atherosclerosis Society; and International Association for the Study of obesity. Circulation. 2009;120:1640-5.

30. American Diabetes Association. Classification and Diagnosis of Diabetes: Standards of Medical Care in Diabetes-2019. Diabetes Care. 2019;4:S13-28.

31. Whelton PK, Carey RM, Aronow WS, Casey DE Jr, Collins KJ, Dennison Himmelfarb C, et al. 2017 ACC/AHA/AAPA/ABC/ACPM/AGS/APhA/ASH/ ASPC/NMA/PCNA guideline for the prevention, detection, evaluation, and management of high blood pressure in adults: a report of the American College of Cardiology/American Heart Association Task Force on Clinical Practice Guidelines. Circulation. 2018;138:e484-594.

32. Turesson C, O'Fallon WM, Crowson CS, Gabriel SE, Matteson EL. Extraarticular disease manifestations in rheumatoid arthritis: incidence trends and risk factors over 46 years. Ann Rheum Dis. 2003;62:722-7.

33. Fransen J, Creemers MC, Van Riel PL. Remission in rheumatoid arthritis: agreement of the disease activity score (DAS28) with the ARA preliminary remission criteria. Rheumatology (Oxford). 2004;43(10):1252-5.

34. Duru N, van der Goes MC, Jacobs JW, Andrews T, Boers M, Buttgereit F, et al. EULAR evidence-based and consensus-based recommendations on the management of medium to high-dose glucocorticoid therapy in rheumatic diseases. Ann Rheum Dis. 2013;72:1905-13.

35. Kerola AM, Kerola T, Kauppi MJ, Kautiainen H, Virta LJ, Puolakka K, Nieminen TV. Cardiovascular comorbidities antedating the diagnosis of rheumatoid arthritis. Ann Rheum Dis. 2013;72:1826-9.

36. Kerola AM, Kauppi MJ, Kerola T, Nieminen TV. How early in the course of rheumatoid arthritis does the excess cardiovascular risk appear? Ann Rheum Dis. 2012;71:1606-15.

37. Kitas GD, Gabriel SE. Cardiovascular disease in rheumatoid arthritis: state of the art and future perspectives. Ann Rheum Dis. 2011;70:8-14.

38. Arts EE, Fransen J, den Broeder AA, Popa CD, van Riel PL. The effect of disease duration and disease activity on the risk of cardiovascular disease in rheumatoid arthritis patients. Ann Rheum Dis. 2015;74:998-1003.

39. Ursini F, Ruscitti P, Caio GPI, Manfredini R, Giacomelli R, De Giorgio R. The effect of non-TNF-targeted biologics on vascular dysfunction in rheumatoid arthritis: a systematic literature review. Autoimmun Rev. 2019;18:501-9.

40. Ursini F, Leporini C, Bene F, D'Angelo S, Mauro D, Russo E, De Sarro G, Olivieri I, Pitzalis C, Lewis M, Grembiale RD. Anti-TNF-alpha agents and endothelial function in rheumatoid arthritis: a systematic review and metaanalysis. Sci Rep. 2017;7:5346.

41. Chung CP, Oeser A, Raggi P, Gebretsadik T, Shintani AK, Sokka T, Pincus T, Avalos I, Stein CM. Increased coronary-artery atherosclerosis in rheumatoid arthritis: relationship to disease duration and cardiovascular risk factors. Arthritis Rheum. 2005;52:3045-53.

42. Ruscitti P, Cipriani P, Masedu F, Romano S, Berardicurti O, Liakouli V, et al. Increased cardiovascular events and subclinical atherosclerosis in rheumatoid arthritis patients: 1 year prospective single centre study. PLoS One. 2017;12:e0170108

43. Provan SA, Semb AG, Hisdal J, Stranden E, Agewall S, Dagfinrud H, et al. Remission is the goal for cardiovascular risk management in patients with rheumatoid arthritis: a cross-sectional comparative study. Ann Rheum Dis. 2011;70:812-7

44. Burggraaf B, van Breukelen-van der Stoep DF, de Vries MA, klop B, Liem $A H$, van de Geijn GM, et al. Effect of a treat-to-target intervention of cardiovascular risk factors on subclinical and clinical atherosclerosis in rheumatoid arthritis: a randomised clinical trial. Ann Rheum Dis. 2019; 78:335-41.

45. Arts EE, Fransen J, Den Broeder AA, van Riel PLCM, Popa CD. Low disease activity (DAS28 3.2 ) reduces the risk of first cardiovascular event in rheumatoid arthritis: a time-dependent Cox regression analysis in a large cohort study. Ann Rheum Dis. 2017;76:1693-9.

46. Barra LJ, Pope JE, Hitchon C, Boire G, Schieir O, Lin D, et al. The effect of rheumatoid arthritis-associated autoantibodies on the incidence of cardiovascular events in a large inception cohort of early inflammatory arthritis. Rheumatology (Oxford). 2017;56:768-76

47. del Rincón I, Polak JF, O'Leary DH, Battafarano DF, Erikson JM, Restrepo JF, et al. Systemic inflammation and cardiovascular risk factors predict rapid progression of atherosclerosis in rheumatoid arthritis. Ann Rheum Dis. 2015;74:1118-23.

48. Cambridge G, Acharya J, Cooper JA, Edwards JC, Humphries SE. Antibodies to citrullinated peptides and risk of coronary heart disease. Atherosclerosis, 2013:228:243-6.
49. Sokolove J, Brennan MJ, Sharpe O, Lahey LJ, Kao AH, Krishnan E, et al. Brief report: citrullination within the atherosclerotic plaque: a potential target for the anti-citrullinated protein antibody response in rheumatoid arthritis. Arthritis Rheum. 2013;65:1719-2.

50. Solow EB, Yu F, Thiele GM, Sokolove J, Robinson WH, Pruhs ZM, et al. Vascular calcifications on hand radiographs in rheumatoid arthritis and associations with autoantibodies, cardiovascular risk factors and mortality. Rheumatology (Oxford). 2015;54:1587-95.

51. Ruscitti P, Ursini F, Cipriani P, Ciccia F, Liakouli V, Carubbi F, et al. Prevalence of type 2 diabetes and impaired fasting glucose in patients affected by rheumatoid arthritis: Results from a cross-sectional study. Medicine (Baltimore). 2017;96:e7896.

52. Ruscitti $P$, Ursini F, Cipriani $P$, Liakouli V, Carubbi F, Berardicurti $O$, et al. Poor clinical response in rheumatoid arthritis is the main risk factor for diabetes development in the short-term: a 1-year, single-centre, longitudinal study. PLoS One. 2017;12:e0181203.

53. Giacomelli R, Ruscitti P, Alvaro S, Ciccia F, Liakouli V, Di Benedetto P, et al. IL-1 $\beta$ at the crossroad between rheumatoid arthritis and type 2 diabetes: may we kill two birds with one stone? Expert Rev Clin Immunol. 2016;12:849-55.

54. Ruscitti P, Cipriani P, Di Benedetto P, Liakouli V, Berardicurti O, Carubbi F, et al. Monocytes from patients with rheumatoid arthritis and type 2 diabetes mellitus display an increased production of interleukin (IL)-1 $\beta$ via the nucleotide-binding domain and leucine-rich repeat containing family pyrin 3(NLRP3)-inflammasome activation: a possible implication for therapeutic decision in these patients. Clin Exp Immunol. 2015:182:35-44.

55. Ursini F, Russo E, Ruscitti P, Giacomelli R, De Sarro G. The effect of non-TNFtargeted biologics and small molecules on insulin resistance in inflammatory arthritis. Autoimmun Rev. 2018;17:399-404.

56. Bartoloni E, Alunno A, Gerli R. Hypertension as a cardiovascular risk factor in autoimmune rheumatic diseases. Nat Rev Cardiol. 2018;15:33-44.

57. Stavropoulos-Kalinoglou A, Metsios GS, Koutedakis Y, Kitas GD. Obesity in rheumatoid arthritis. Rheumatology (Oxford). 2011;50:450-62.

58. Choy E, Ganeshalingam K, Semb AG, Szekanecz Z, Nurmohamed M. Cardiovascular risk in rheumatoid arthritis: recent advances in the understanding of the pivotal role of inflammation, risk predictors and the impact of treatment. Rheumatology (Oxford). 2014;53:2143-54.

59. Yusuf S, Hawken S, Ounpuu S, Dans T, Avezum A, Lanas F, et al. Effect of potentially modifiable risk factors associated with myocardial infarction in 52 countries (the INTERHEART study): case-control study. Lancet. 2004;364:937-52.

60. Póliska S, Besenyei T, Végh E, Hamar A, Pusztai A, Váncsa A, et al. Gene expression analysis of vascular pathophysiology related to anti-TNF treatment in rheumatoid arthritis. Arthritis Res Ther. 2019;21:94.

61. Bissell LA, Erhayiem B, Fent G, Hensor EMA, Burska A, Donica H, et al. Carotid artery volumetric measures associate with clinical ten-year cardiovascular (CV) risk scores and individual traditional CV risk factors in rheumatoid arthritis; a carotid-MRI feasibility study. Arthritis Res Ther. 2018;20:266.

62. Chandrasekharan UM, Wang Z, Wu Y, Wilson Tang WH, Hazen SL, Wang S, et al. Elevated levels of plasma symmetric dimethylarginine and increased arginase activity as potential indicators of cardiovascular comorbidity in rheumatoid arthritis. Arthritis Res Ther. 2018;20:123.

\section{Publisher's Note}

Springer Nature remains neutral with regard to jurisdictional claims in published maps and institutional affiliations.

\section{Ready to submit your research? Choose BMC and benefit from:}

- fast, convenient online submission

- thorough peer review by experienced researchers in your field

- rapid publication on acceptance

- support for research data, including large and complex data types

- gold Open Access which fosters wider collaboration and increased citations

- maximum visibility for your research: over $100 \mathrm{M}$ website views per year

At $\mathrm{BMC}$, research is always in progress.

Learn more biomedcentral.com/submissions 